\title{
Scleroedema of Buschke
}

\section{An uncommon cause of cardiomyopathy}

\author{
M. L. Johnson and H. Ikram \\ From the Departments of Dermatology and Medicine, Charing Cross Hospital, London W.C.2
}

A case of scleroedema of Buschke involving skin and myocardium in a 69-year old woman is described. Widespread electrocardiographic abnormalities were present at the height of the disease, which improved over 18 months as the mucopolysaccharide infiltration of the skin cleared.

Scleroedema is an uncommon disease primarily affecting the skin which becomes infiltrated by acid mucopolysaccharides. The onset of the illness commonly follows an upper respiratory infection and the disease tends to be self-limiting, the skin infiltration gradually clearing in a few months (Rook, 1954; Curtis and Shulak, 1965).

While in the great majority the skin alone is affected, there have been a few cases reported in which there has been systemic involvement.

This condition must not be confused with the more common 'scleroderma', which is a collagen disorder and also has systemic manifestations including myocardial involvement. In this condition, in contrast to scleroedema, the skin collagen becomes hypertrophied and condensed, and cardiac involvement, when it does occur, usually takes the form of an irreversible myocardial fibrosis.

We report a case of scleroedema in which there was evidence of myocardial involvement which subsequently resolved pari passu with clearing of the skin. This represents an unusual and potentially recoverable form of cardiomyopathy.

\section{Case report}

The patient was a 69-year-old housewife who developed a severe upper respiratory tract infection for which she was given a course of tetracycline. Two weeks later, she noticed a tense swelling of the skin of her face, neck, forearms, and hands. The swelling spread to the upper part of the chest and back. She was otherwise completely well. There was no significant illness in her past medical history, and in particular there was no angina or dyspnoea and her exercise tolerance was normal.

On examination there was tense induration of the skin of the face, neck (Fig. I), and the upper part of the chest and back. The infiltration was initially diffuse, but became more papular on the neck over the following weeks; the accentuated follicles gave a peau d'orange appearance. Similar, less obvious changes were present in the antecubital fossae and on the medial surface of the arms. The affected skin did not pit on pressure. The remainder of the skin including the scalp was normal. Cardiological opinion: no orthopnoea. Pulse 85 a minute, normal. Neck veins not visible because of skin infiltration. No sacral or ankle oedema. Heart sounds normal. Grade 2 ejection systolic murmur in aortic area. Blood pressure was $130 / 80 \mathrm{~mm}$. $\mathrm{Hg}$. Remainder of clinical examination normal.

Histology of skin: excess acid mucopolysaccharides present.

Investigations Skiagram of chest. Slight cardiac enlargement. $\mathrm{C} / \mathrm{T}$ ratio $\mathbf{1} 4 \cdot 5 / 25$. Lung fields clear. Electrocardiogram (Fig. 2). Widespread ST-T wave abnormality consistent with nonspecific myocardial damage. Right atrial pressure $5 / 0 \mathrm{~mm}$. Hg. No evidence of cardiac compression on the venous pressure pulse. Right ventricular pressure $30 / 0 \mathrm{~mm}$. $\mathrm{Hg}$. All pressures measured with zero level at sternal angle. Haemoglobin 98 per cent. White blood cells 6,000/cu. mm. Normal differential count. Erythrocyte sedimentation rate $8 \mathrm{~mm}$./hr. (Westergren). Serum protein $6.3 \mathrm{~g}$. $/$ IOO $\mathrm{ml}$. Electrophoretic pattern, normal.

Serum aspartate aminotransferase and serum alanine aminotransferase were normal. Lactic acid dehydrogenase 1230 units (normal 300-500 units), suggestive of cardiac involvement. Serum cholesterol $195 \mathrm{mg} \cdot 100 \mathrm{ml}$. Protein-bound iodine $4.6 \mu \mathrm{g}$./ $100 \mathrm{ml}$. Tri-iodo thyronine resin uptake 99 per cent of normal. 
FIG. I Left, neck showing peau d'orange due to infiltration; right, I8 months after onset showing normal texture of skin.

Viral antibody titres Para-influenza 3 on 23 October 1967 I/I60; on 6 November 1967 I/320, suggesting preceding Para-influenza 3 infection.

Progress She was treated symptomatically with simple emollient creams. The skin improved slowly and after a year there was no obvious thickening at any site. Eighteen months after the onset, the skin was normal (Fig. I) and the electrocardiogram showed significant improvement (Fig. 2). The chest skiagram was unchanged.

\section{Discussion}

Scleroedema was first described under this name by Buschke in 1902. The disease commonly follows an upper respiratory infection, usually streptococcal. Women are affected twice as often as men, and approximately 50 per cent of cases present before and 50 per cent after the age of 20. Skin involvement consists of progressive infiltration of the dermis by acid mucopolysaccharides (Fleischmajer and Lara, 1965), with a resultant woody and tense induration of the skin which starts on the back and sides of the neck and spreads to the face, arms, and upper chest. The skin changes usually clear in a few months, but may persist for up to two years or longer (Curtis and Shulak, 1965).

Vallee (1946) reported a 37-year-old woman with scleroedema who had a pericardial effusion which persisted for a year. This was associated with low voltage QRS complexes and flat $T$ waves. The cutaneous and cardiac abnormalities disappeared at the same time.

Cardiac involvement was also reported by Leinwand (195I) who described a 58-year-old woman who developed exertional dyspnoea 5 years after the onset of cutaneous scleroedema and who died one year later from progressive myocardial failure with widespread ST depression and $T$ wave inversion. Necropsy showed a pericardial effusion and a rubbery rigidity of the ventricular muscle. The coronary arterial tree was normal.

There have been other cases in which transient myocardial involvement occurred. Robinow (1963) reported two children with scleroedema who developed tachycardia and gallop rhythm with $T$ wave inversion and prolongation of the QT interval on the electrocardiogram. These changes reverted to normal in three months. Curtis and Shulak (1965) described inversion of the $T$ wave in $V_{2}$ and terminal inversion in $V_{3}$ and $V_{4}$ which persisted for 3 months in a 16-year-old girl with the disease.

The evidence of cardiac involvement in our case consists of the widespread $T$ wave 
inversion which resolved pari passu with the skin changes. The cardiographic changes were too widespread to be due to localized ischaemic changes. Moreover, she had no clinical evidence of ischaemic heart disease. The improvement of the electrocardiogram in a 70year-old woman suggested that these changes were not due to degenerative causes expected at this age.

In the only necropsy reported (Leinwand, 195I) the myocardial fibres showed splitting, suggesting interstitial infiltration. No staining for acid mucopolysaccharides was carried out so the nature of the infiltrate remains a matter for speculation, but it seems likely that it is similar to the acid mucopolysaccharide infiltrate in the skin.

There is a similarity between the changes in this condition and myxoedema in which there is a mucinous infiltration of the skin and heart resulting in $T$ wave changes and small voltage QRS complexes.

From the earlier case reports, it is evident that cardiac involvement in scleroedema may manifest itself as a pericardial effusion or as infiltration of the myocardium. It may vary in severity from being asymptomatic to causing death from intractable heart failure. The evidence of cardiac failure, post-mortem evidence of myocardial degeneration, and prolongation of the QT interval and gallop rhythm (Robinow, 1963) are evidence that the electrocardiographic changes reflect heart involvement rather than merely being due to changes in skin conductivity as a result of the dermatological lesion. It is important to recognize this condition because it usually has a good prognosis, unlike most other forms of cardiomyopathy which are frequently fatal.

We are grateful to Dr. O. L. S. Scott for permission to report this case and to the Cardiographic Department of the Charing Cross Hospital for the electrocardiography. The dermatological aspects were discussed at the Dermatology Section of the Royal Society of Medicine at the onset of the disease (Johnson, Ikram and Scott, 1968).

\section{References}

Curtis, A. C., and Shulak, B. M. (1965). Scleredema adultorum. Not always a benign self-limited disease. Archives of Dermatology, 92, 526.

Fleischmajer, R., and Lara, J. V. (1965). Scleredemaa histochemical and biochemical study. Archives of Dermatology, 92, 643 .

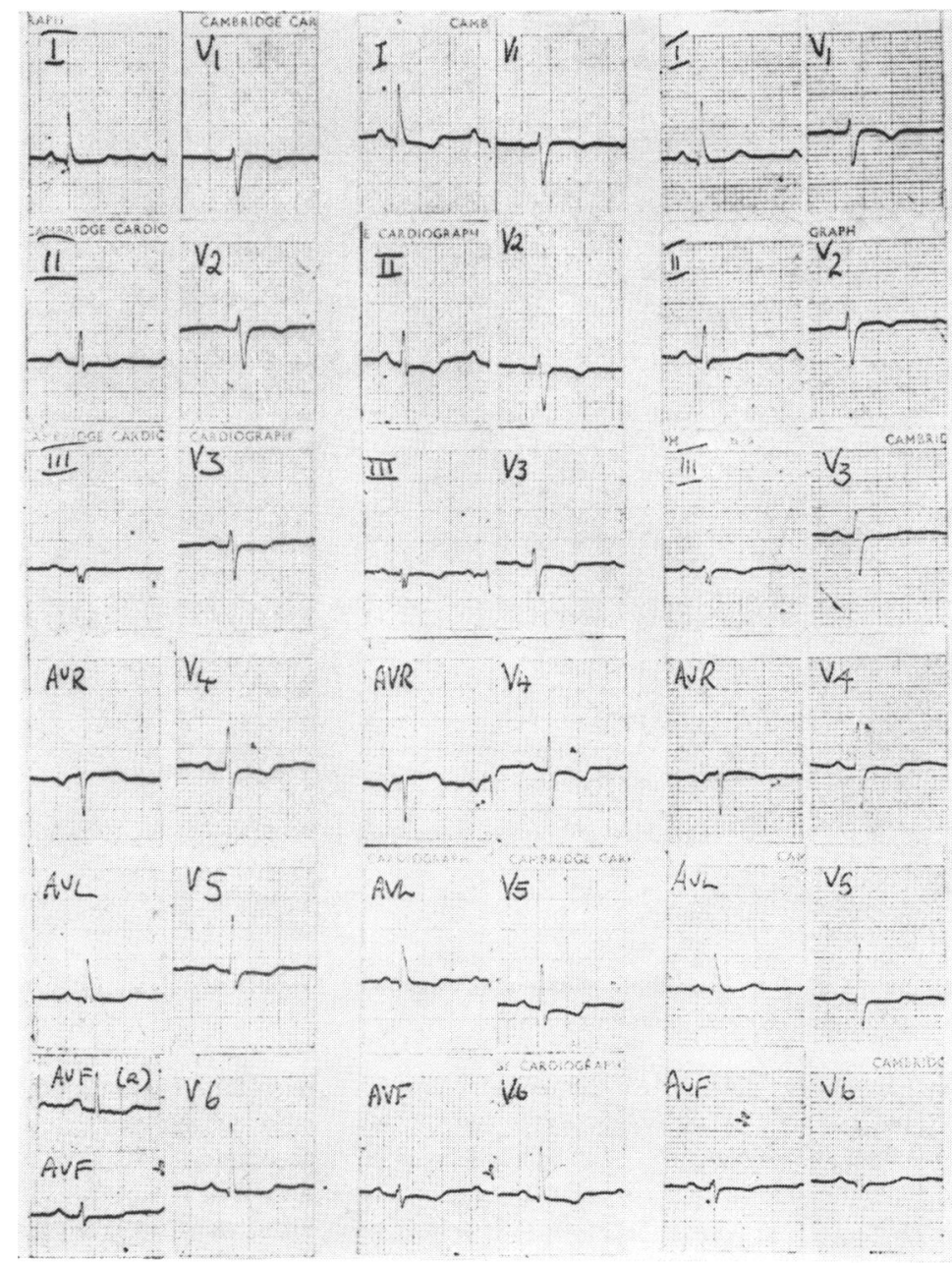

FIG. 2 Serial electrocardiograms showing increasing abnormalities in the early stage of the disease, with subsequent improvement as the skin returned to normal.

Johnson, M. L., Ikram, H., and Scott, O. L. S. (1968). Scleroedema (Buschke) with cardiac involvement. British fournal of Dermatology, 80, 196.

Leinwand, I. (I95I). Generalized scleredema: Report with autopsy findings. Annals of Internal Medicine, 34, 226.

Robinow, M. (1963). Scleredema adultorum-a children's disease. American Fournal of Diseases of Children, r05, 265.

Rook, A. (1954). Scleroedema. Postgraduate Medical fournal, 30, 30.

Vallee, B. L. (1946). Scleredema: A systemic disease. New England fournal of Medicine, 235, 207. 